\title{
Elevated Tribbles homolog 2-specific antibody levels in narcolepsy patients
}

\author{
Vesna Cvetkovic-Lopes,, Laurence Bayer, ${ }^{1}$ Stéphane Dorsaz, ${ }^{2}$ Stéphanie Maret, ${ }^{2}$ \\ Sylvain Pradervand, ${ }^{3}$ Yves Dauvilliers, ${ }^{4}$ Michel Lecendreux, ${ }^{5}$ Gert-Jan Lammers, ${ }^{6}$ \\ Claire E.H.M. Donjacour, ${ }^{6}$ Renaud A. Du Pasquier, 7,8 Corinne Pfister, ${ }^{2}$ Brice Petit, ${ }^{2}$ \\ Hyun Hor, ${ }^{2,4}$ Michel Mühlethaler, ${ }^{1}$ and Mehdi Tafti ${ }^{2,9}$
}

\begin{abstract}
1Département de Neurosciences Fondamentales, Centre Médical Universitaire, Genève, Switzerland. ${ }^{2}$ Center for Integrative Genomics, University of Lausanne, Switzerland. ${ }^{3}$ Lausanne DNA Array Facility, University of Lausanne. ${ }^{4}$ Department of Neurology, Hôpital Gui de Chauliac, CHU Montpellier, Inserm U888, Montpellier, France. ${ }^{5}$ Child and Adolescent Psychopathology Unit, Robert Debré Hospital, Paris VII University, Paris, France. ${ }^{6}$ Department of Neurology and Clinical Neurophysiology, Leiden University Medical Centre, Netherlands. ${ }^{7}$ Division of Immunology and Allergy, ${ }^{8}$ Division of Neurology, and ${ }^{9}$ Center for Investigation and Research in Sleep, Centre Hospitalouniversitaire Vaudois, Lausanne.
\end{abstract}

\begin{abstract}
Narcolepsy is a sleep disorder characterized by excessive daytime sleepiness and attacks of muscle atonia triggered by strong emotions (cataplexy). Narcolepsy is caused by hypocretin (orexin) deficiency, paralleled by a dramatic loss in hypothalamic hypocretin-producing neurons. It is believed that narcolepsy is an autoimmune disorder, although definitive proof of this, such as the presence of autoantibodies, is still lacking. We engineered a transgenic mouse model to identify peptides enriched within hypocretin-producing neurons that could serve as potential autoimmune targets. Initial analysis indicated that the transcript encoding Tribbles homolog 2 (Trib2), previously identified as an autoantigen in autoimmune uveitis, was enriched in hypocretin neurons in these mice. ELISA analysis showed that sera from narcolepsy patients with cataplexy had higher Trib2-specific antibody titers compared with either normal controls or patients with idiopathic hypersomnia, multiple sclerosis, or other inflammatory neurological disorders. Trib2-specific antibody titers were highest early after narcolepsy onset, sharply decreased within 2-3 years, and then stabilized at levels substantially higher than that of controls for up to 30 years. High Trib2-specific antibody titers correlated with the severity of cataplexy. Serum of a patient showed specific immunoreactivity with over $86 \%$ of hypocretin neurons in the mouse hypothalamus. Thus, we have identified reactive autoantibodies in human narcolepsy, providing evidence that narcolepsy is an autoimmune disorder.
\end{abstract}

\section{Introduction}

Hypocretin (orexin) neurons play a critical role in the regulation of sleep and wakefulness, and disturbances of the hypocretin system have been directly linked to narcolepsy in animals and humans (1-6). Human narcolepsy is believed to be caused by a selective hypocretin neuronal loss $(2,3)$. Current hypotheses suggest an autoimmune process targeting these neurons. Attempts to characterize immune-related processes have failed so far. Narcolepsy is tightly associated with the HLA system, with $95 \%$ of narcolepsy patients with cataplexy carrying the HLADQB1*0602 allele and having undetectable hypocretin levels in their cerebrospinal fluid (CSF) $(7,8)$. A recent genome-wide association study found a strong association between narcolepsy and a $T$ cell receptor $\alpha$ gene variant, corroborating the autoimmune hypothesis (9). Moreover, using a model of spontaneous colonic migrating motor complex, the presence of functional autoantibodies in sera of narcolepsy patients could be demonstrated (10, 11). However, peripheral or central immune abnormalities in narcolepsy, even in patients diagnosed shortly after the disease onset, could not be demonstrated thus far (12). One hypothesis is that hypocretin neurons express a specific peptide recognized as an autoantigen. The autoimmune attack may be acute and

Authorship note: Vesna Cvetkovic-Lopes and Laurence Bayer, as well as Michel Mühlethaler and Mehdi Tafti, contributed equally to this work.

Conflict of interest: Mehdi Tafti received research funding from UCB Pharma S.A., Belgium.

Citation for this article: J Clin Invest. 2010;120(3):713-719. doi:10.1172/JCI41366. narcolepsy symptoms may develop once hypocretin neurons are lost, with autoantibody titers below detectable levels. Neuronal pentraxin 2 (NPTX2 or Narp) and prodynorphin (PDYN), two peptides that are colocalized with hypocretin within the posterior lateral hypothalamus, were, like hypocretin, absent in the few postmortem brains of narcolepsy patients (13). However, because a putative immune attack does not seem to target either hypocretin ligands or their receptors (12) and because Narp and PDYN are abundantly expressed in many brain structures, they are unlikely autoantigen targets. To screen for hypocretin-coexpressed peptides that may be targets of an autoimmune attack in narcolepsy, we have engineered a transgenic mouse model. We show here that Tribbles homolog 2 (Trib2) transcript is enriched over 3 fold in hypocretin-producing neurons. Because Trib2 was found as an autoantigen in autoimmune uveitis (14), we have developed an ELISA assay to test for the presence of autoantibodies against Trib2 in sera and CSF of narcolepsy patients, control subjects, and other neurological patients. Surprisingly, we found that narcolepsy patients have high titers of circulating autoantibodies against Trib2 as compared with all other groups. Moreover, we show that serum of a narcolepsy patient showed immunoreactivity with over $86 \%$ of hypocretin neurons in the mouse hypothalamus and that preabsorption of the serum with recombinant Trib2 peptide abolished immunoreactivity. These results indicate for the first time to our knowledge that Trib2 is an autoantigen in human narcolepsy and that Trib2-specific antibodies specifically target hypocretin neurons, ultimately leading to their disappearance and hypocretin deficiency. 


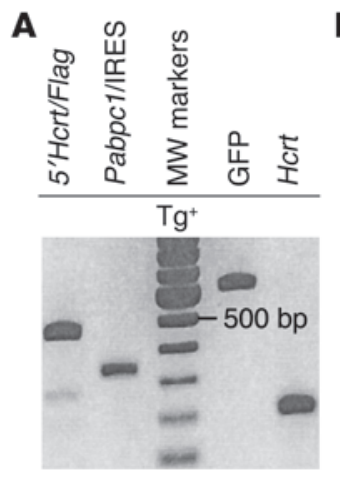

B
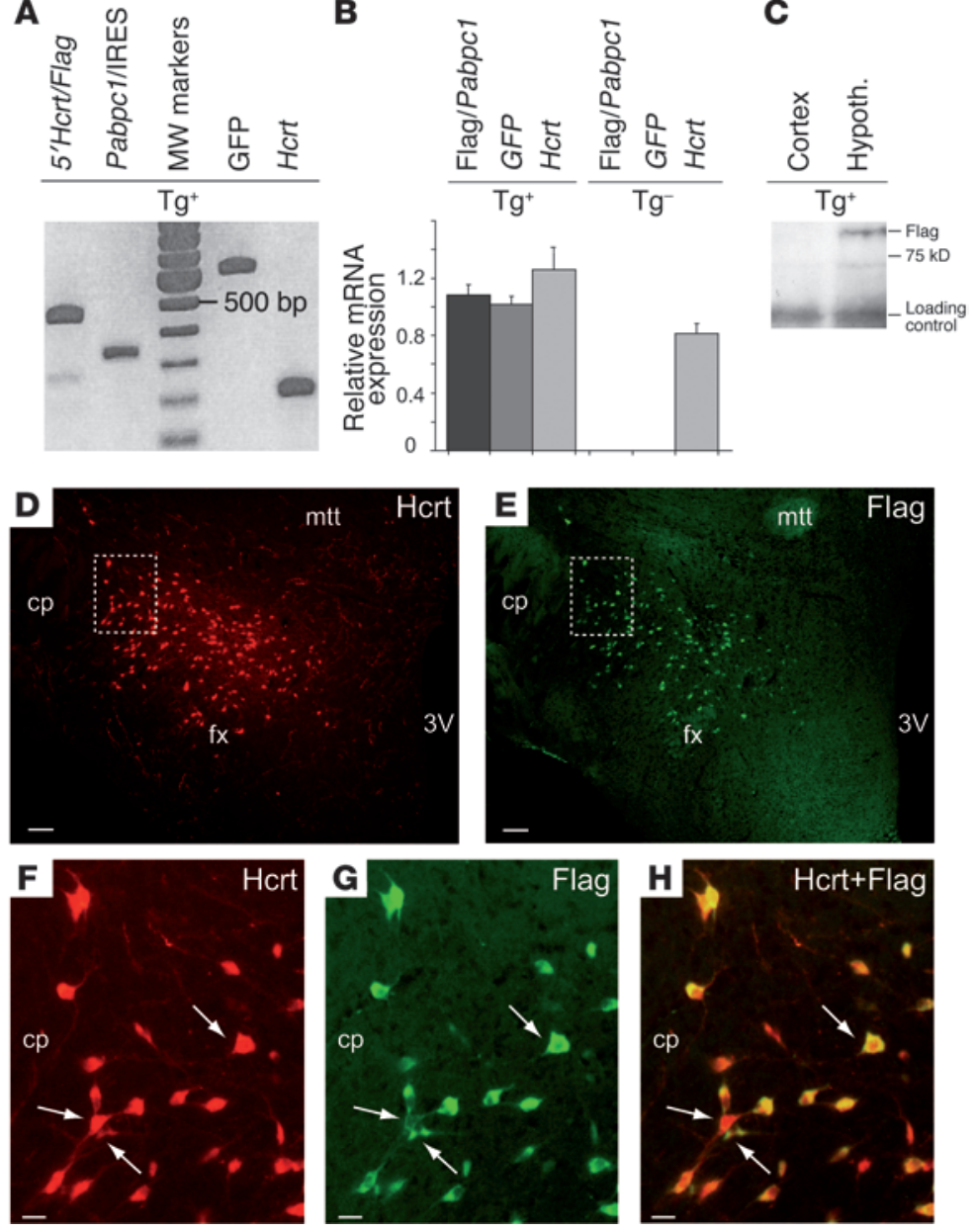

\section{Figure 1}

Pabpc1-hypocretin transgenic mice. (A) RT-PCR experiments performed on total RNAs from a transgenic mouse hypothalamus $(\mathrm{Tg}+)$, showing the presence of both the construct and hypocretin (Hcrt) transcripts. Four different primer pairs were used, each generating a PCR fragment of the size predicted by its cDNA sequence: 5' Hcrt/Flag (467 bp), Pabpc1/IRES (333 bp), GFP (611 bp), and Hcrt (189 bp). (B) Quantitative RT-PCR experiments showing similar relative RNA expression levels for Flag-tagged Pabpc1, GFP, and Hcrt in transgenic mice hypothalamus. Only the Hcrt transcript was detected in nontransgenic (Tg-) mouse hypothalamus as expected. The normalized relative values (+1SD) obtained correspond to the mean values from 4 biological replicates per condition. (C) Western blot analysis demonstrating the presence of the Flag protein in the hypothalamic (hypoth.) extract (obtained from 3 transgenic mice) and its absence in the cortical fraction (from the same 3 mice). The loading control is a nonspecific band generated by the mouse monoclonal anti-Flag M2 antibody. Photomicrographs illustrating the distribution of (D and $\mathbf{F}$ ) Hcrt- and ( $E$ and $\mathbf{G}$ ) Flag-expressing cells on a coronal section from the tuberal hypothalamus. $(\mathbf{F}-\mathbf{H})$ Higher magnification views of the dashed regions in $\mathbf{D}$ and $\mathbf{E}$ confirm that Hcrt and Flag are coexpressed in the same neurons. Arrows indicate both single Hcrt or Flag or double Hcrt-Flag staining. Almost all Hcrt neurons were found to express the Flag-containing construct. (H) Merged image of $\mathbf{F}$ and $\mathbf{G}$. $\mathrm{cp}$, cerebral peduncle; fx, fornix; mtt, mammilo-thalamic tract; $3 \mathrm{~V}$, third ventricle. Scale bar: $100 \mu \mathrm{m}$ (D and E); $20 \mu \mathrm{m}(\mathbf{F}-\mathbf{H})$.

\section{Results}

$m R N A$ tagging and identification from bypocretin-producing neurons. To identify proteins coexpressed with hypocretin, we generated transgenic mice to profile gene expression in hypocretin neurons. In a BAC-based construct, we replaced the hypocretin coding sequence with a Flag-tagged poly(A)-binding protein (Pabpc1) cDNA sequence (see Methods and Supplemental Figure 1; supplemental material available online with this article; doi:10.1172/JCI41366DS1). This construct takes advantage of the ability of Pabpc1 to bind the poly(A) tails of mRNAs in vivo. Thus, only mRNAs from hypocretin cells are expected to cross-link with the Flag-tagged Pabpc1, which can then be captured by coimmunoprecipitating with a specific anti-flag monoclonal antibody (15). Two transgenic lines were obtained and one was fully characterized as follows. RT-PCR experiments from hypothalamic total RNA extracts detected the presence of a Flag, a Pabpc1, and an EGFP fragment in transgenic mice (Figure 1A). Similar relative expression levels of Flag, EGFP, and hypocretin (Hcrt) mRNAs were found by real-time RT-PCR in transgenic hypothalami, whereas only Hcrt mRNA was detected in nontransgenic mice (Figure 1B). A monoclonal mouse anti-Flag antibody detected presence of the Flag protein in mouse hypothalamic protein but not in cortical extracts (Figure 1C). The specific cellular expression of the Flag-Pabpc1 construct was also demonstrated by double immunofluorescence. All Flag-expressing cells were hypocretin positive, and all hypocretinexpressing neurons were Flag positive (Figure 1, D-H).
Melanin-concentrating hormone $(P m c h)$ and hypocretin neurons form 2 distinct but partially overlapping populations in the hypothalamus. To assess mRNA tagging efficacy, we compared the ratio of Hcrt mRNA level recovered to that of Pmch in transgenic IP samples and in transgenic whole-brain RNA samples (Supplemental Figure 2). Consistent with our expectations, Hcrt mRNA expression was enriched 20 fold after IP. Applying this protocol to nontransgenic mouse brains did not result in hypocretin enrichment.

Comparison between IP mRNA and total brain mRNA $(n=9)$ in transgenic mice using Affymetrix Mouse Genome Array indicated that 1,721 probe sets were enriched at least 3 fold in IP samples (false discovery rate [FDR] <5\%; Supplemental Table 1). Fold enrichment ranged between 3 and 107. Many of the highly enriched transcripts turned out to be unannotated or matched several genomic localizations. However, probe set 1447461_at was enriched 30 fold and mapped to an expressed sequence (LOC100048678) with high homology with Spindlins, which are meiotic spindle-binding proteins involved in cell cycle. Overexpression of Spindlin1 (Spin1) was recently found to induce cellular senescence, multinucleation, and apoptosis (16). Another highly enriched probe set (1420300_at; 16.5-fold) indicated GABA-A2 receptor subunit gene (Gabra2). Although Gabra2 mRNA has been detected in hypocretin-producing neurons, Gabra2 protein was reported not to be expressed (17). 
A

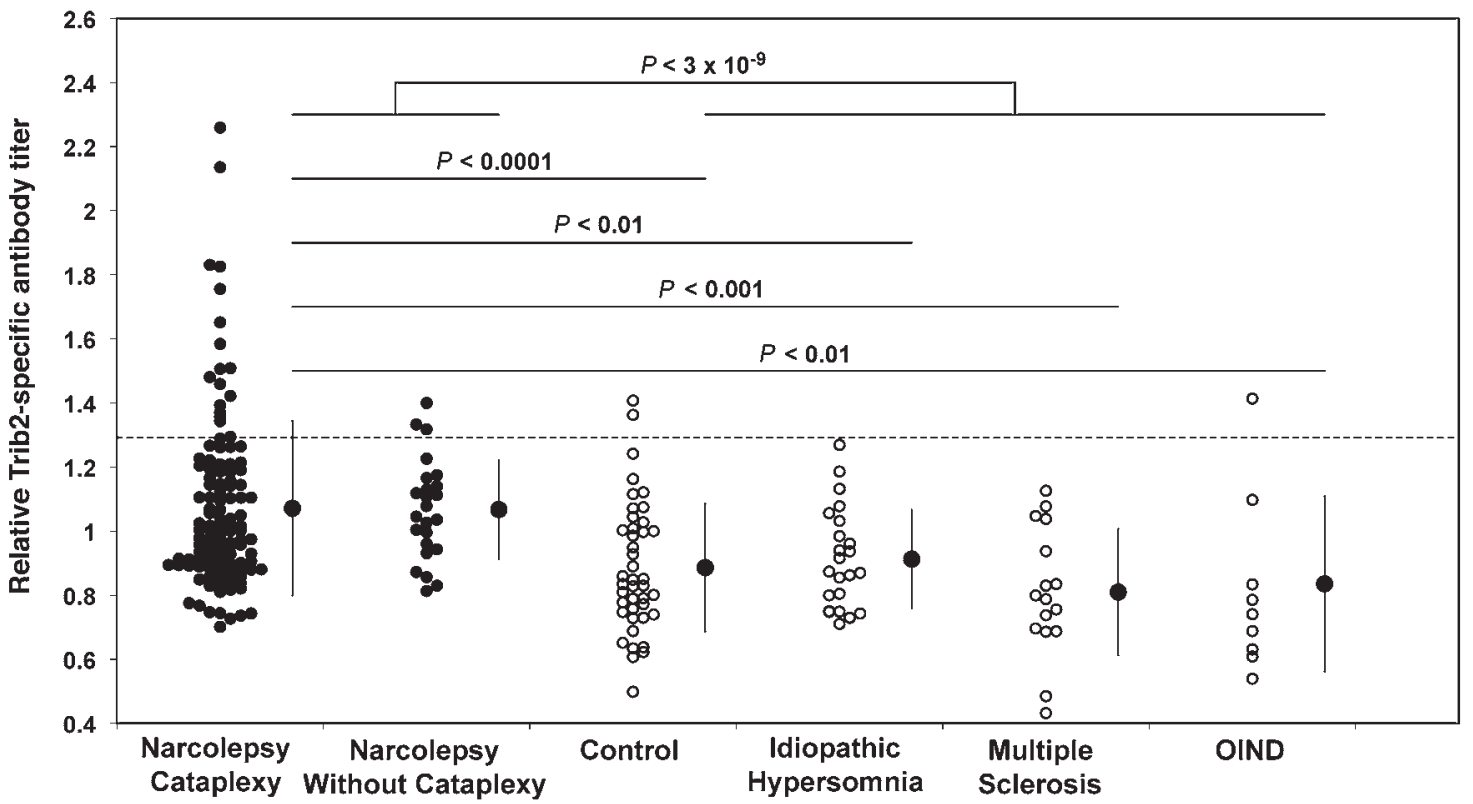

B

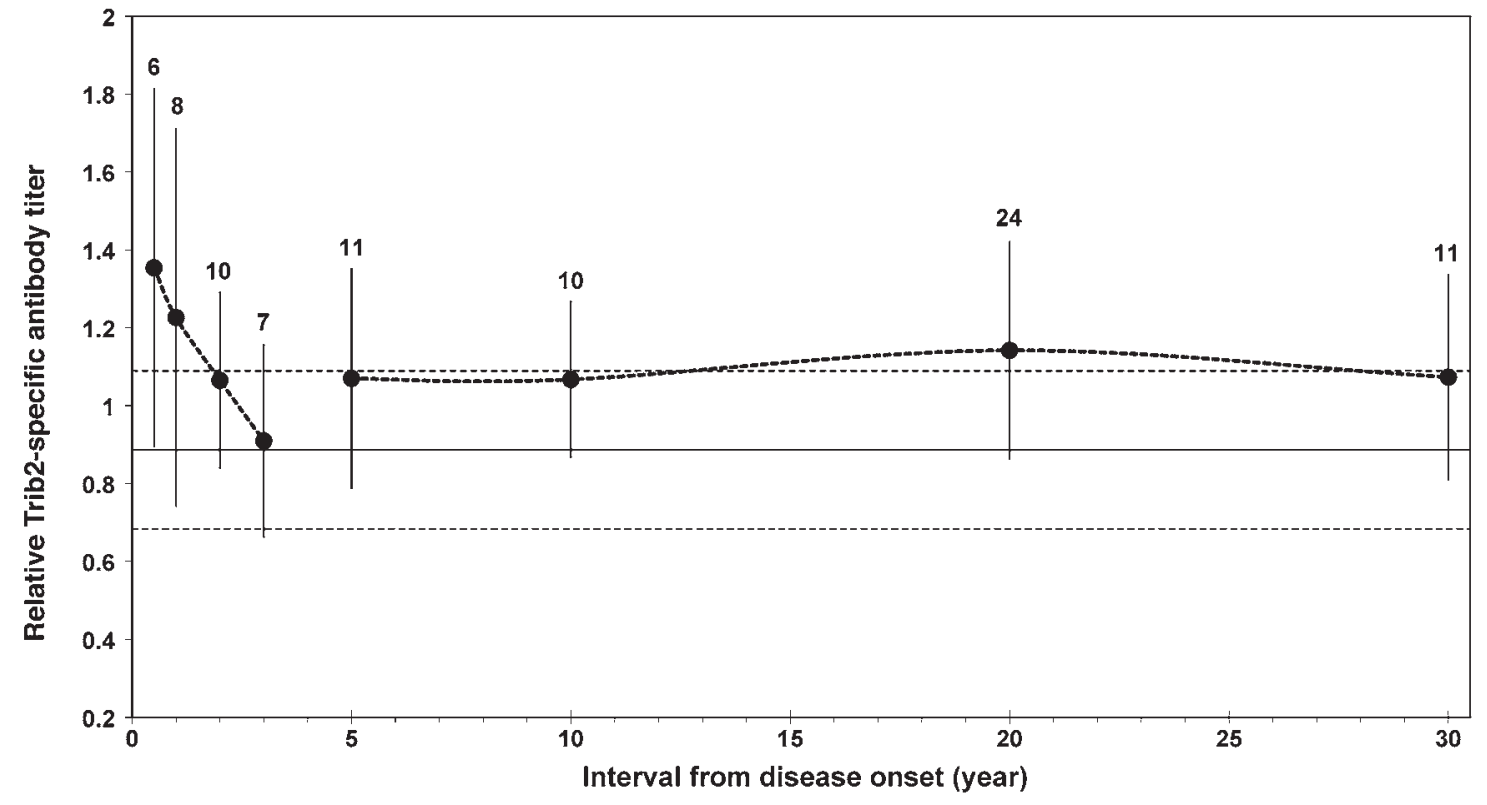

Figure 2

ELISA determination of Trib2-specific antibodies in sera. (A) Each symbol corresponds to the serum of a single subject. Mean \pm 1 SD of each group is shown next to the individual values. The dotted horizontal line indicates the mean Trib2-specific antibody titer in healthy control subjects plus 2 SD. All values are relative to the optical density of a healthy control subject (which is equal to 1). $P$ values correspond to independent $t$-tests between indicated groups. OIND, other inflammatory neurological diseases. (B) Mean \pm 1 SD of Trib2-specific antibody titers at different intervals from the disease onset. The solid line and dotted lines indicate mean titer \pm 1 SD in normal control subjects. Numbers indicate the number of narcolepsy patients at each interval. Note the sharp decrease in titers within the first 2-3 years, reaching normal values. From 5 up to 30 years after disease onset, the titers of narcolepsy patients remain stable but significantly $\left(1 \mathrm{SD}, P<5 \times 10^{-5}\right)$ higher than those of healthy control subjects $(n=42)$.

A recent study investigated mRNA expression in the hypothalamic hypocretin region in mice and in postmortem human narcolepsy samples and identified insulin-like growth factor binding protein 3 (Igfbp3) as a potential colocalized protein (18). In the present study, Igfbp3 was unchanged or slightly depleted from IP mRNA samples (probe set 1423062_at; fold change, -1.2; FDR, 0.02). Of the 2 peptides reported to be colocalized with hypocretin, Narp transcript was indeed slightly enriched in IP samples (probe set 1420720_at; fold change, 2.15; FDR, $<5.10^{-6}$ ), while prodynorphin was not (probe set 1416266_at; fold change, -1.1; FDR, 0.48).

To identify the most specific genes expressed in hypocretinproducing neurons, we selected those probe sets among the 1,721 that were 3 -fold enriched and called present in at least 7 microarrays hybridized with IP mRNA and called absent in at least 7 microarrays hybridized with total brain RNA. This analysis yielded only 23 probe sets (Supplemental Table 2). None of these 23 

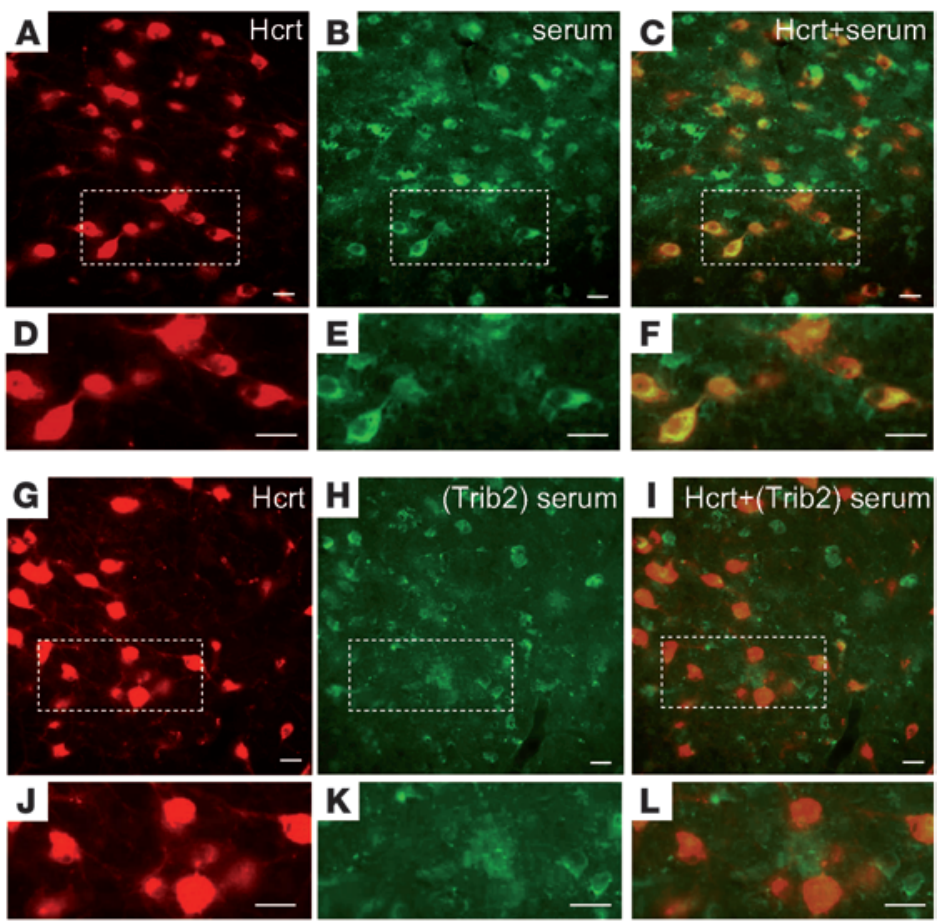

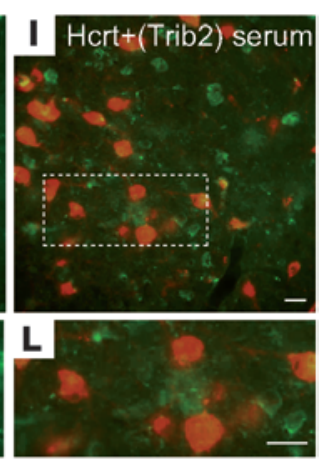

\section{Figure 3}

Double immunohistochemistry with hypocretin antibody and a high Trib2-specific antibody-containing serum of a narcolepsy with cataplexy patient. (A) Coronal section through the mouse hypothalamus, stained with anti-hypocretin antibody. (B) The same section stained with the patient's serum. (C) Merged hypocretin-serum staining showing that the vast majority of hypocretin-positive neurons are costained by the patient's serum. (D-F) Higher magnification views of the dashed regions in A-C confirm the double labeling of hypocretin neurons. Overall, $87 \%$ of hypocretin neurons are costained by the serum. (G) Another mouse hypothalamic section stained with anti-hypocretin antibody. (H) Immunoreactivity with the same patient's serum as in B but after preabsorption of Trib2-specific antibodies with excess Trib2 peptide. (I) Merged image showing that the vast majority of hypocretin neurons are only stained with hypocretin antibody. Only $8 \%$ of hypocretin neurons are double labeled. (J-L) Higher magnification views of the dashed regions in $\mathbf{G}-\mathbf{I}$ confirm that serum depletion of Trib2-specific antibodies results in the absence of double staining. Scale bar: $20 \mu \mathrm{m}$. transcripts were reported to be specifically expressed in hypocretin neurons, based on the mouse brain atlas (Allen Institute for Brain Science; http://mouse.brain-map.org/). Nevertheless, among these genes, several are of functional importance and might be involved in abnormal functioning of hypocretin neurons in narcolepsy. Four of such candidate genes (Igf $2 b p 2$, Slc12a6, Spin1, and Trib2) were tested and, except for Spin1, confirmed by quantitative PCR. Trib2 was the only gene found to be an autoantigen in an autoimmune disorder, uveitis (14). Intermediate uveitis was found to be associated with HLA-DR15 and narcolepsy in a patient (19). Also one of our narcolepsy patients was affected with uveitis. To explore this further, we developed an ELISA assay to detect Trib2-specific autoantibodies in sera and CSF of narcolepsy patients.

ELISA assay detects Trib2-specific autoantibodies in narcolepsy patients. A glutathione $S$-transferase-tagged fragment containing the last 28 amino acids of Trib2 (14) was used as a target, and all 96-well assays were run with serum of a normal subject for normalization. Sera from 119 narcolepsy with cataplexy patients (HLA-DQB1*0602 positive) were run at 1:100 dilution against sera of 24 narcolepsy without cataplexy patients, 42 normal subjects, 23 idiopathic hypersomnia patients, 16 multiple sclerosis patients, and 9 other patients with neurological inflammatory diseases. Relative Trib2-specific antibody titers were significantly increased in the narcolepsy with cataplexy group as compared with all other groups (Figure 2A) except for narcolepsy without cataplexy. When contrasting narcolepsy patients to all others, the relative Trib2-specific antibody titers were highly significantly increased $\left(P<3 \times 10^{-9}\right)$. Fifty-six out of one hundred forty-three narcolepsy patients (39\%) had titers higher than the mean titer of the controls (>1 SD) and 20 (14\%) had titers more than 2 SD above the mean titer of healthy controls. Even with the restrictive 2-SD criterion more narcolepsy patients were positive for Trib2-specific antibodies than all other groups (20 narcolepsy patients with $[n=17]$ and without cataplexy $[n=3]$ versus 3 healthy controls $[n=2]$ and patients with other inflammatory neurological diseases $[n=1]$;
Fisher's exact test, $P<0.03$ ). Trib2-specific antibody titers of HLA DQB1*0602-positive and -negative subjects were not significantly different. In addition to all narcolepsy with cataplexy patients who were DQB $1 * 0602$ positive, HLA typing was available in 27 out of 42 controls (64\%), 18 out of 23 idiopathic hypersomnia patients (78\%), and 19 out of 24 narcolepsy without cataplexy patients (79\%), and overall $13(15 \%)$ were HLA-DQB1*0602 positive (4 controls and 4 idiopathic hypersomnia and 5 narcolepsy without cataplexy patients) but only one narcolepsy without cataplexy patient had Trib2-specific antibody titers higher than 2 SD above the mean of the healthy control group, strongly suggesting that high Trib2-specific antibody titers are specific to narcolepsy. In 96 narcolepsy with cataplexy patients, the delay between the disease onset and the time of serum withdrawal could be precisely established. Although we did not find a correlation between Trib2-specific antibody titers and the duration of disease, among the 17 high Trib2-specific antibody titer (>2 SD) narcolepsy with cataplexy patients, $5(29 \%)$ were sampled within the first year of disease onset against 9 (11\%) out of the remaining 79 with lower titers $\left(\chi^{2}=3.65, P<0.06\right)$. Trib2-specific antibody titers sharply decreased within the first 2-3 years after disease onset (Figure 2B) but remained $1 \mathrm{SD}$ above the mean titer of healthy controls, even 30 years after disease onset $\left(P<5 \times 10^{-5}\right)$. In 10 out of the 17 patients with greater than 2 SD titers, complete clinical and laboratory data were available. Trib2-specific antibody titers were significantly and positively correlated with the frequency of cataplexy (Spearman rank correlation, $r=0.74, P<0.02)$ and tended to be correlated with the severity of sleepiness (Epworth sleepiness scale, $r=0.67, P<0.06$ ). CSF samples were available in a limited number of patients (26 narcolepsy with cataplexy, 6 narcolepsy without cataplexy, 4 idiopathic hypersomnia, and 15 multiple sclerosis patients). Trib2-specific antibody could be detected at low levels without a significant difference among groups. Nevertheless, in 18 narcolepsy with cataplexy patients, paired serum-CSF samples could be assessed, and a positive correlation was found $(r=0.54, P<0.03)$. 
Trib2-specific autoantibodies target bypocretin neurons. To show that hypocretin-producing neurons are a target of Trib2-specific antibodies, mouse hypothalamic sections were double stained with a serum of a narcolepsy with cataplexy patient (age 8 , serum available 10 months after disease onset) with high Trib2-specific antibody titer and an anti-hypocretin antibody. Immunoreactivity against the patient's serum was found throughout the hypothalamus but, more specifically, over $86 \%$ of hypocretin neurons were double labeled (Figure 3, A-F). To confirm the specificity of the immunoreactivity with hypocretin neurons, the same serum was depleted of Trib2-specific antibodies by preincubation with excess 28 amino acid Trib2 peptide. Depleted serum showed dramatic decrease (less than $8 \%$ ) in hypocretin neuron staining (Figure 3, G-I), confirming the presence of Trib2-specific autoantibodies targeting hypocretin neurons in the patient's serum. The same experiments with a healthy control serum or the serum from our narcolepsy with uveitis patient (interval of disease onset for more than 15 years, and Trib2-specific antibody titer was within the normal range) did not show any specific staining (between $50 \%$ to $60 \%$ of hypocretin neurons showed nonspecific staining before and after preadsorption, respectively).

\section{Discussion}

These findings indicate for the first time the presence of identified and autoreactive antibodies in human narcolepsy. High Trib2-specific antibody titers (>2.5-3 SD) were found in narcolepsy with cataplexy patients exclusively, but we did not find an overall difference between narcolepsy with and without cataplexy. Furthermore, antibody titers correlate both with the severity of cataplexy and sleepiness, suggesting that the 2 conditions might share a similar pathophysiology. Note that hypocretin deficiency is also found in up to $20 \%$ of narcolepsy without cataplexy patients and a recent study found $30 \%$ hypocretin neuron loss in a narcolepsy without cataplexy patient (20), corroborating our finding that Trib2-specific antibodies might target hypocretin neurons in both groups but at lesser extend in narcolepsy without cataplexy.

Although neither Trib2 nor any other peptide was found specific to hypocretin neurons, given the low level of Trib2 expression, but its specific enrichment in hypocretin neurons, it may be that in addition to other sporadic neurons, hypocretin neurons are the major target of the autoimmune attack, resulting primarily in narcolepsy symptoms. Nevertheless, other colocalized and/or enriched transcripts, such as Igf $2 b p 2$ or Slc12a6, might play some functional role in hypocretinergic neurotransmission, as is also suggested for Igfbp3 (18). Trib2-specific antibodies seem to predominate in narcolepsy patients, with recent disease onset further suggesting that these antibodies are pathogenic. Although only $14 \%$ of all our narcolepsy patients had Trib2-specific antibody titers over 2 SD above controls, $30 \%$ of patients with an interval from disease onset of less than 1 year had titers greater than 2 SD. Trib2-specific antibody titers sharply decreased within the first 2-3 years of disease onset but remained significantly higher than controls up to 30 years, suggesting that narcolepsy might be triggered by an acute autoimmune process and not a recurrent process as in many other autoimmune disorders. Together with HLA and T cell receptor $\alpha$ associations, our findings strongly suggest that narcolepsy can be an autoimmune disorder.

Obviously not all narcolepsy patients have high Trib2-specific antibody titers even close to the disease onset, suggesting more complex mechanisms leading to the development of the condi- tion. The time course of a potential autoimmune process targeting hypocretin neurons may vary from patient to patient. Some patients may have a rapid course with major deficit in hypocretin production, resulting in sudden appearance of excessive daytime sleepiness and cataplexy, while others may have a slower course, resulting in excessive daytime sleepiness followed, up to several years later, by cataplexy when the number of hypocretin neurons reaches a critical value. It may also be that Trib2 is not the only autoantigen and/or not the most specific one. As in other autoimmune disorders (e.g., type 1 diabetes), several autoantibodies may be involved but not all are detectable in the majority of patients. Finally, although we have showed here for the first time that an autoimmune reaction can be detected, this does not imply that autoimmunity is involved in all forms of narcolepsy.

We have reported several cases of narcolepsy treated with intravenous immunoglobulins (IVIg) soon after the first symptoms with unexpected positive results, suggesting that the autoimmune process may be counteracted if treated early $(21,22)$. Most recently, we have reported a narcolepsy patient with hypocretin deficiency, in whom the CSF hypocretin level normalized after IVIg treatment (23). Our present findings strengthen the autoimmune hypothesis and suggest that patients with an early diagnosis and high Trib2specific antibody titers might benefit from immunotherapy.

\section{Methods}

Transgenic mice production, $m R N A$ tagging, and IP. BAC-based Flag-tagged Pabpc1-hypocretin transgenic mice were generated following a procedure (Supplemental Figure 1) similar to that described for the generation of Homer1a-Pabpc1 transgenic mice (15). The study protocol was approved by the Lausanne Veterinary Office.

Our mRNA tagging process was modified from 2 previously described procedures in Caenorhabditis elegans and Drosophila $(24,25)$ and was demonstrated by us to successfully enrich Homerla-specific mRNAs in HomerlaPabpc1 transgenic mice (15).

RT-PCR and real-time RT-PCR experiments. RT-PCR genotyping of transgenic mice was performed with primers specific for the construction (Pabpc1/IRES forward 5'-AAGCCATGCACCCTTCTCTTGC-3', reverse 5'-CCTTATTCCAAGCGGCTTCGG-3'; GFP forward 5'-GAGCTGGACGGCGACGTAAACG-3', reverse 5'-AGGACCATGTGATCGCGCTTCTC-3'). Quantitative RT-PCR experiments to detect endogenous hypocretin, our construct, and candidate genes were performed by TaqMan Universal PCR Master Mix (Applied Biosystems) in 384-well plates in an ABI PRISM 7900 detection system (Applied Biosystems). Following standard procedures, gene-specific primers and TaqMan probes were as follows: Hypocretin TaqMan gene expression assay (Applied Biosystems), Pmch forward, 5'-AGAGATTTTGACATGCTCAGGTGTA-3', reverse 5'-GCGGACCAGCAGGTATCAGA-3', probe 5'-CTGGGAAGAGTCTACCGACCATGTTGGC-3'; flag-Pabpc1 forward 5'-TAAAGATCATGACATCGATTACAAGGA-3', reverse 5'-AGGTCCCCCACGTACAGAGA-3', probe 5'-ACGATGACAAGCTCGACAT-3'; GFP forward 5'CTGCTGCCCGACAACCAC-3', reverse 5'-ACCATGTGATCGCGCTTCTC3', probe, 5'-CCAGTCCGCCCTGAGCAAAGACC-3' (all from Eurogentec).

Western blotting analysis. Three transgenic mice were rapidly sacrificed and coronal brain slices (300- $\mu \mathrm{m}$ thick) were cut on a Vibratome in artificial CSF. Slices containing hypocretin neurons were selected and microdissected with a razor blade in order to keep only the hypothalamic region. All hypothalamicdissected regions were pooled together and protein extract was prepared with RIPA lysis buffer on ice. Cortex punches from each slice were also collected, pooled together, and similarly treated as a control extract. Protein concentration was calculated by using Bradford assay and $32 \mu \mathrm{g}$ of each fraction were separated on a SDS-PAGE and transferred to a Nitrocellulose membrane. 
After 1 hour of blocking in Tween/Tris-buffered saline and 5\% non-fat milk, membranes were incubated overnight with the monoclonal mouse anti-flag M2 primary antibody (Sigma-Aldrich) diluted 1:300, followed by 1 hour with the secondary HRP-conjugated goat anti-mouse antibody diluted 1:6,000, and revealed using ECL reagents and Hyperfilm ECL.

Double immunohistochemistry for hypocretin and Flag. Paraformaldehydefixed brains were serially cut into $35-\mu \mathrm{m}$ coronal floating slices on a cryostat. Sections, taken at $140-\mu \mathrm{m}$ intervals throughout the whole hypothalamus, were processed for a double immunofluorescence procedure. The Flag staining was first revealed with the mouse monoclonal anti-flag M2 antibody diluted 1:500, followed by 2 hours with the Alexa Fluor 488-conjugated goat anti-mouse IgG1 secondary antibody diluted 1:1,000. After rinsing in PBS, the hypocretin labeling was performed with a rabbit anti-hypocretin A serum diluted 1:2,000, followed by Alexa Fluor 555 -conjugated goat anti-rabbit IgG secondary antibody diluted 1:1,000. Sections were observed with a Zeiss Axioskop 2 microscope (filter sets 10, $15)$ equipped with an AxioCam color CCD camera (Zeiss). Images were recorded on computer through the AxioVision software (Zeiss). A confirmation survey on a confocal microscope was also performed.

Microarray experiments. Targets were prepared from either $5 \mathrm{ng}$ IP mRNAs or 5 ng of whole brain total RNAs, using the NuGen WT-Ovation Pico RNA amplification system. The generated cDNAs $(5 \mu \mathrm{g})$ were fragmented, biotinylated, and hybridized using the NuGen FL-Ovation cDNA Biotin Module V2, following the manufacturer's instructions. The resulting targets were hybridized to Affymetrix GeneChip Mouse Genome 2302.0 arrays. All statistical analysis were performed using the free high-level interpreted statistical language R (The R Project for Statistical Computing, R Core, 2004; http://www.R-project.org) and various Bioconductor packages (http://www.Bioconductor.org). Hybridization quality was assessed using Bioconductor "affy" and "affyPLM" packages $(26,27)$. Because of the differences between expression profiles of IP and whole brain samples, normalized expression signals were calculated in Affymetrix Expression Console using MAS 5.0, which simply scales the overall expression signal to the same intensity. Differential hybridized features were identified from all probe sets using Bioconductor package "limma" on log2 transformed MAS 5.0 normalized values (28). $P$ values were adjusted for multiple testing with Benjamini and Hochberg's method to control the FDR. Probe sets showing at least 3 -fold change and a FDR less than 0.05 were considered significant. Data are available at the Gene Expression Omnibus database (accession number GSE17617; http://www.ncbi.nlm.nih.gov/geo).

Subjects. Narcolepsy with cataplexy, narcolepsy without cataplexy, and idiopathic hypersomnia patients were included based on diagnostic criteria of the International Classification of Sleep Disorders (ICSD-2). The frequency of cataplexy was assessed by a scale from 1 to 5 , reporting rare to very frequent cataplexy attacks (29). Healthy controls were recruited in affiliated sleep centers and had full clinical and, in more than half, polysomnography investigations. Affiliated neurology departments recruited multiple sclerosis and other inflammatory neurological patients. All participants gave written informed consent and the ethics committee of the Faculty of Biology and Medicine of the University of Lausanne approved the study protocol.

ELISA. A GST fusion protein containing $28 \mathrm{C}$-terminal amino acids (NSAYGAKEVSDQLVPDVNMEENLDPFFN) of Trib2 was expressed and purified (14). The vector containing the recombinant peptide was a gift of W. Li (University of Miami Miller School of Medicine, Florida). Trib2- specific antibody quantifications in narcolepsy patients and controls were determined using ELISA standard procedures. Ten $\mu \mathrm{g}$ of GST-TRIB2 partial fusion protein were deposited in 96-well ELISA plates and incubated overnight at $4{ }^{\circ} \mathrm{C}$. Wells were washed and blocked for 1 hour at room temperature with $1 \%$ BSA. One hundred $\mu$ l of diluted serum (1:100) or $50 \mu \mathrm{l}$ of CSF were applied in triplicates for 1 hour at room temperature. Anti-human IgG-biotin (diluted 1:10,000) and streptavidin-HRP (diluted $1: 10,000)$ were then consecutively incubated 1 hour at room temperature. Colorimetric assay was performed, using O-phenylenediamine diluted in citrate-phosphate buffer, and $30 \% \mathrm{H}_{2} \mathrm{O}_{2}$ was added just before use. After 10 minutes, the reaction was stopped with $3 \mathrm{~N} \mathrm{HCL}$ and absorbance was read at $492 \mathrm{~nm}$. For normalization and interplate calibration, 3 serum samples were repetitively quantified on each plate (intra-assay coefficient of variation, $<6 \%$; inter-assay coefficient of variation, $<15 \%$ ). At least 10 sera were tested both against GST-18 amino acid Trib2 or GST-full-length Trib2 with virtually identical results, strongly suggesting that the partial peptide contains an epitope recognized by autoantibodies.

Double immunohistochemistry for bypocretin and narcolepsy serum. Immunochemistry was performed on fixed floating $35-\mu \mathrm{m}$ mouse brain coronal sections. Sections were incubated for 24 hours at room temperature, with a high Trib2-specific antibody-containing serum of a narcolepsy with cataplexy patient (dilution at 1:2). They were rinsed in PBS and incubated for 2 hours at room temperature with an anti-human IgG-biotin antibody (dilution at 1:1,000), followed by 2 hours of incubation with Cy2-conjugated streptavidin (dilution at 1:1,000). A double immunostaining was performed to reveal hypocretin neurons with a rabbit anti-hypocretin A serum (dilution at 1:1,000), followed by Alexa Fluor 555-conjugated anti-rabbit IgG secondary antibody (dilution at 1:1,000). To demonstrate specificity of Trib2 labeling with narcolepsy serum, the same procedure was repeated with the narcolepsy serum preabsorbed during 2 hours with $18 \mathrm{C}$-terminal amino acids of Trib2, at the concentration of $4 \mu \mathrm{g} / \mathrm{ml}$.

Statistics. Microarray gene expression analysis used standard statistical methods explained above. Antibody titers were compared by 2 -tailed Student's $t$ test. Other tests included Fisher exact test, $\chi^{2}$, Spearman rank correlation, and Pearson's correlation, and $P$ values of less than 0.05 were considered significant.

\section{Acknowledgments}

We thank patients for their involvement in the study; Yann Emmenegger, Keith Harshman, Otto Hagenbuchle, and Alexandra Paillusson for technical assistance; and Paul Franken and Anne Vassalli for critical reading of our manuscript. This work was supported by the Swiss National Science Foundation grants 3100A0120726 to M. Mühlethaler and 3100A0-108478 to M. Tafti.

Received for publication October 7, 2009, and accepted in revised form January 6, 2010.

Address correspondence to: Mehdi Tafti, Center for Integrative Genomics (CIG), University of Lausanne, Génopode, 1015 Lausanne, Switzerland. Phone: 4121.692.3971; Fax: 4121.692.3965; E-mail:mtafti@unil.ch.

Stéphanie Maret's present address is: Department of Psychiatry, University of Wisconsin, Madison.
1. Dauvilliers Y, Arnulf I, Mignot E. Narcolepsy with cataplexy. Lancet. 2007;369(9560):499-511.

2. Thannickal TC, et al. Reduced number of hypocretin neurons in human narcolepsy. Neuron. 2000;27(3):469-474.

3. Peyron C, et al. A mutation in a case of early onset narcolepsy and a generalized absence of hypocretin peptides in human narcoleptic brains. Nat Med. 2000;6(9):991-997.

4. Willie JT, et al. Distinct narcolepsy syndromes in Orexin receptor- 2 and Orexin null mice: molecular genetic dissection of Non-REM and REM sleep regulatory processes. Neuron. 2003;38(5):715-730.

5 . Hara J, et al. Genetic ablation of orexin neurons in mice results in narcolepsy, hypophagia, and obesity. Neuron. 2001;30(2):345-354.

6. Nishino S, Ripley B, Overeem S, Lammers GJ, 
Mignot E. Hypocretin (orexin) deficiency in human narcolepsy. Lancet. 2000;355(9197):39-40.

7. Mignot E, et al. The role of cerebrospinal fluid hypocretin measurement in the diagnosis of narcolepsy and other hypersomnias. Arch Neurol. 2002;59(10):1553-1562.

8. Mignot E. Genetic and familial aspects of narcolepsy. Neurology. 1998;50(2 Suppl 1):S16-S22.

9. Hallmayer J, et al. Narcolepsy is strongly associated with the T-cell receptor alpha locus. Nat Genet. 2009;41(6):708-711.

10. Smith AJ, Jackson MW, Neufing P, McEvoy RD, Gordon TP. A functional autoantibody in narcolepsy. Lancet. 2004;364(9451):2122-2124.

11. Jackson MW, Reed JH, Smith AJ, Gordon TP. An autoantibody in narcolepsy disrupts colonic migrating motor complexes. J Neurosi. 2008;28(49):13303-13309.

12. Overeem S, Black JL 3rd, Lammers GJ. Narcolepsy: immunological aspects. Sleep Med Rev. 2008;12(2):95-107.

13. Crocker A, et al. Concomitant loss of dynorphin, NARP, and orexin in narcolepsy. Neurology. 2005;65(8):1184-1188.

14. Zhang Y, Davis JL, Li W. Identification of tribbles homolog 2 as an autoantigen in autoimmune uveitis by phage display. Mol Immunol.
2005;42(11):1275-1281.

15. Maret S, et al. Homer1a is a core brain molecular correlate of sleep loss. Proc Natl Acad Sci U S A. 2007;104(50):20090-20095.

16. Yuan $\mathrm{H}$, et al. Overexpression of SPINDLIN1 induces cellular senescence, multinucleation and apoptosis. Gene. 2008;410(1):67-74.

17. Backberg M, Ultenius C, Fritschy JM, Meister B. Cellular localization of GABA receptor alpha subunit immunoreactivity in the rat hypothalamus: relationship with neurones containing orexigenic or anorexigenic peptides. J Neuroendocrinol. 2004;16(7):589-604.

18. Honda M, et al. IGFBP3 colocalizes with and regulates hypocretin (orexin). PLoS ONE. 2009;4(1):e4254.

19. Tang WM, Pulido JS, Eckels DD, Han DP, Mieler WF, Pierce K. The association of HLADR15 and intermediate uveitis. Am J Ophthalmol. 1997;123(1):70-75.

20. Thannickal TC, Nienhuis R, Siegel JM. Localized loss of hypocretin (orexin) cells in narcolepsy without cataplexy. Sleep. 2009;32(8):993-998.

21. Lecendreux M, Maret S, Bassetti C, Mouren MC, Tafti M. Clinical efficacy of high-dose intravenous immunoglobulins near the onset of narcolepsy in a 10-year-old boy. J Sleep Res. 2003;12(4):347-348.

22. Dauvilliers Y, Carlander B, Rivier F, Touchon J,
Tafti M. Successful management of cataplexy with intravenous immunoglobulins at narcolepsy onset. Ann Neurol. 2004;56(6):905-908.

23. Dauvilliers Y, Abril B, Mas E, Michel F, Tafti M. Normalization of hypocretin-1 in narcolepsy after intravenous immunoglobulin treatment. Neurology. 2009;73(16):1333-1334.

24. Roy PJ, Stuart JM, Lund J, Kim SK. Chromosomal clustering of muscle-expressed genes in Caenorhabditis elegans. Nature. 2002;418(6901):975-979.

25. Yang Z, Edenberg HJ, Davis RL. Isolation of mRNA from specific tissues of Drosophila by mRNA tagging. Nucleic Acids Res. 2005;33(17):e148.

26. Bolstad BM, Irizarry RA, Astrand M, Speed TP. A comparison of normalization methods for high density oligonucleotide array data based on variance and bias. Bioinformatics. 2003;19(2):185-193.

27. Gautier L, Cope L, Bolstad BM, Irizarry RA. affy-analysis of Affymetrix GeneChip data at the probe level. Bioinformatics. 2004;20(3):307-315.

28. Smyth GK. Linear models and empirical bayes methods for assessing differential expression in microarray experiments. Stat Appl Genet Mol Biol. 2004;3:Article3.

29. Dauvilliers Y, et al. Age at onset of narcolepsy in two large populations of patients in France and Quebec. Neurology. 2001;57(11):2029-2033. 